\title{
Optimization of thin n-in-p planar pixel modules for the ATLAS upgrade at HL-LHC
}

\author{
A. Macchiolo, ${ }^{a, 1}$ J. Beyer, ${ }^{a}$ A. La Rosa, ${ }^{a}$ R. Nisius ${ }^{a}$ and N. Savic ${ }^{a}$ \\ ${ }^{a}$ Max-Planck-Institut für Physik, \\ Föhringer Ring 6, 80805 Munich, Germany \\ E-mail: Anna.Macchiolo@mpp.mpg.de
}

\begin{abstract}
AвSTRACT: The ATLAS experiment will undergo around the year 2025 a replacement of the tracker system in view of the high luminosity phase of the LHC (HL-LHC) with a new 5-layer pixel system. Thin planar pixel sensors are promising candidates to instrument the innermost region of the new pixel system, thanks to the reduced contribution to the material budget and their high charge collection efficiency after irradiation. The sensors of 50-150 $\mu \mathrm{m}$ thickness, interconnected to FEI4 read-out chips, have been characterized with radioactive sources and beam tests. In particular active edge sensors have been investigated. The performance of two different versions of edge designs are compared: the first with a bias ring, and the second one where only a floating guard ring has been implemented. The hit efficiency at the edge has also been studied after irradiation at a fluence of $10^{15} \mathrm{n}_{\mathrm{eq}} / \mathrm{cm}^{2}$. Highly segmented sensors will represent a challenge for the tracking in the forward region of the pixel system at HL-LHC. In order to reproduce the performance of 50x50 $\mu \mathrm{m}^{2}$ pixels at high pseudo-rapidity values, FE-I4 compatible planar pixel sensors have been studied before and after irradiation in beam tests at high incidence angles with respect to the short pixel direction. Results on the hit efficiency in this configuration are discussed for different sensor thicknesses.
\end{abstract}

KeYwords: PIXEL,Radiation-hard detectors; Particle tracking detectors

\footnotetext{
${ }^{1}$ Corresponding author.
} 


\section{Contents}

1 Introduction 1

2 Thin planar pixel sensor productions 1

3 Test beam characterization of active edge sensors 3

4 Tracking performance at high incidence angle 4

5 Conclusions 6

\section{Introduction}

The ATLAS experiment at CERN is planning a complete replacement of the tracking detector around 2024-2026 in view of the High Luminosity phase of the LHC (HL-LHC). The istantaneous luminosity is foreseen to be increased to $5-7 \times 10^{34} \mathrm{~cm}^{-2} \mathrm{~s}^{-1}$ and after ten years of running the integrated luminosity is expected to be around $3000 \mathrm{fb}^{-1}$ [1]. The new pixel system will be composed by 5 layers, with the two innermost ones foreseen to be replaced at half of the Inner Tracker (ITk) lifetime. The maximum fluence expected before replacement in the innermost layer is around 1$1.2 \times 10^{16} \mathrm{n}_{\mathrm{eq}} / \mathrm{cm}^{2}$ [2]. A higher pixel granularity with respect to the $50 \times 400$ and $50 \times 250 \mu \mathrm{m}^{2}$ cell size presently employed in the ATLAS pixel detector [3] will be necessary to cope with the increased particle rate, and the new read-out chip, being developed by the RD53 Collaboration in $65 \mathrm{~nm}$ CMOS technology, will have a pitch of $50 \times 50 \mu \mathrm{m}^{2}$ [4].

In this paper thin planar pixel sensors, based on the n-in-p technology, are investigated as possible candidates to instrument all the layers of the ITk pixel system, and their electrical characterization before and after irradiation is shown in a sensor thickness range between 50 and $150 \mu \mathrm{m}$. The main benefits of the use of thin planar pixel sensors are their high production yield, reduced contribution to the material budget and higher radiation resistance thanks to the higher electric field that can be established in the bulk with respect to thicker sensors at the same applied bias voltage. The radiation resistance of $100 \mu \mathrm{m}$ thin planar sensors has been proven up to a fluence of $10^{16} \mathrm{n}_{\mathrm{eq}} / \mathrm{cm}^{2}$ and a power dissipation of $25-50 \mathrm{~mW} / \mathrm{cm}^{2}$ has been estimated at an operational temperature of $-25^{\circ} \mathrm{C}[5]$.

\section{Thin planar pixel sensor productions}

Different production methods for thin planar pixel sensors are being investigated at the moment, from the established use of Silicon on Insulator (SOI) wafers to a new technology employed at CiS [6] that does not foresee the use of a handle wafer. For all the sensors here described the inter-pixel isolation is achieved by means of a homogeneous p-spray realized with a maskless low dose boron implantation. 

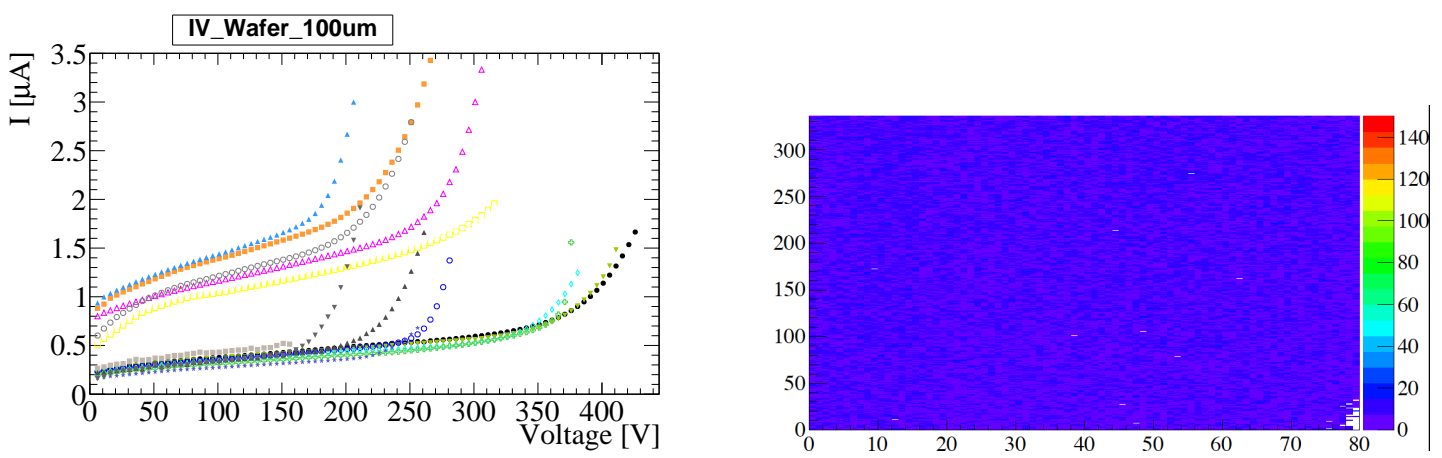

Figure 1. Left side: IV curves measured wtith $100 \mu \mathrm{m}$ thin bare sensors of the CiS production with backside cavities. The sensors are of two different sizes, for single FE-I4 chip or four chip assemblies. The depletion voltage for this thickness is around $10 \mathrm{~V}$. Right side: hit map obtained with a Cadmium source scan on a FE-I4 module (336 rows $\times 80$ columns). The map shows a good uniformity of the number of hits per pixel, except on the bottom right corner where the white color indicates that no hits were recorded.

The thinning technology at CiS is based on anisotropic potassium hydroxide $(\mathrm{KOH})$ etching to create backside cavities in the wafer leaving thicker frames around each single structure. The process, described in more detail in [7] has the potential to be more cost-effective compared to standard techniques involving the use of SOI wafers. The first test has been performed on 4-inch wafers of $525 \mu \mathrm{m}$ starting thickness, where the area below each sensor has been thinned to 100 or $150 \mu \mathrm{m}$. IV curves performed after dicing on $100 \mu \mathrm{m}$ thick sensors are shown in Fig.1, with the breakdown voltages around ten times higher than the depletion voltage of $10 \mathrm{~V}$ for these devices. This first production has also been used to investigate two different Under Bump Metallization (UBM) technologies at CiS, involving thin film deposition of Nickel-Gold or Platinum layers as post-processing steps to allow for solder adhesion to the pixel Aluminum pads. These UBM methods are both compatible with the SnAg bump technology for the read-out chip wafers, developed at the Fraunhofer Institute IZM. Modules with the two UBM types, flip-chipped at IZM [8], have been investigated by means of scans with radioactive sources and beam tests at CERN-SPS. The homogenous number of hits measured per channel with a $\mathrm{Cd}$ source scan, shown in the right plot of Fig.1, hints to a very high interconnection yield. Given the good results obtained with this 4-inch wafer production, including mainly FE-I4 compatible sensors, a second one, on 6-inch wafers, has been started to realize sensors to be interconnected to the RD53A prototype chip, with pixel cell sizes of $50 \times 50 \mu \mathrm{m}^{2}$ and $25 \times 100 \mu \mathrm{m}^{2}$.

An alternative method to produce thin sensors is the use of SOI wafers where the backside support is etched away at the end of the processing of the front-side. With this technology a production at ADVACAM [9] employing 6-inch Float Zone (FZ) wafers was organized to investigate planar n-in-p pixel sensors with active edges. Three different sensor thicknesses have been chosen: 50,100 and $150 \mu \mathrm{m}$. Trenches are realized around the perimeter of each sensor with Deep Reactive Ion Etching (DRIE) using the mechanical support offered by the handle wafer of the SOI stack. A boron implantation is performed in the trenches with a four-quadrant ion implantation [10], allowing to extend the depleted volume up to the edges. After the UBM processing, the handle wafer is removed, singularizing the structures. Also in this case, inter-pixel isolation is achieved by mean of 
a homogeneous p-spray. A slim edge and an active edge design have been implemented differing by the distance of the last pixel implant to the sensor edge, indicated as $d$ in the following. The slim edge version with $\mathrm{d}=100 \mu \mathrm{m}$ (Fig.2 (a)), includes a bias ring and a punch-through biasing structure. The active edge design (Fig.2 (b)) characterized by $\mathrm{d}=50 \mu \mathrm{m}$ includes only one floating guard ring (GR). No punch-through structure is implemented in this design.

(a)

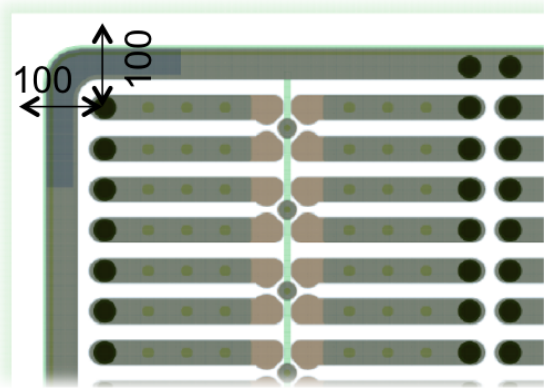

(b)

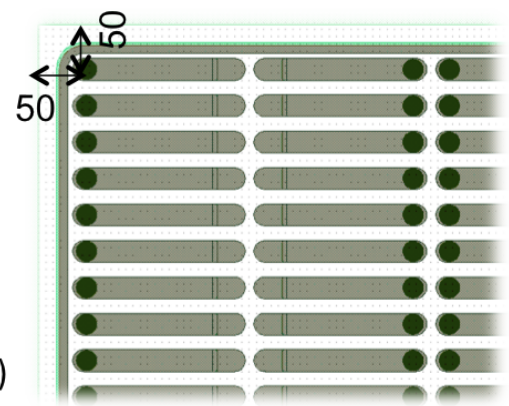

Figure 2. Left: Slim edge design with $100 \mu \mathrm{m}$ distance between the last pixel implant and the edge, it implements a bias ring and punch-through structures. Right: Active edge design with $50 \mu \mathrm{m}$ distance between the last pixel implant and the edge, only a floating bias ring is included.

Results of a first electrical characterization of all sensor types after flip-chipping to FE-I4 chips are summarized in Fig.3 and show a good performance, in particular for the case of the 50 $\mu \mathrm{m}$ thin sensors, where the depletion voltage has been measured to be lower than $10 \mathrm{~V}$. The IV curves on the left plot indicate typical breakdown voltages above $100 \mathrm{~V}$, and very low levels of the leakage current, around $10 \mathrm{nA} / \mathrm{cm}^{2}$. The hit map in the right plot, obtained by means of a Cadmium source scan with a $50 \mu \mathrm{m}$ thick sensor, hints to a very low level of disconnected channels, given the homogenous hit counts per pixel. The measurements presented in this paper are based on devices with an electroplated CuAu UBM, that resulted in a better performance in terms of noise and interconnection yield with respect to thin film UBM that was also investigated with active edge sensors from the same wafers.

\section{Test beam characterization of active edge sensors}

The performance of the active edge sensors has been investigated with beam tests at CERN-SPS by using telescopes of the EUDET family [11]. Fig.4 shows a comparison of the hit efficiency measured at a beam test at CERN-SPS with not-irradiated active edge sensors of different thickness. It can be observed that while the edge in the 100 and $150 \mu \mathrm{m}$ thick sensors is almost fully efficient, the $50 \mu \mathrm{m}$ thick sensor is only efficient up to the end of the last pixel implant. Moreover also in this area the hit efficiency is $97 \%$, lower than for the thicker sensors, with the threshold tuned to a nominal value of $800 \mathrm{e}^{-}$. Further measurements are being carried out to determine the efficiency with an optmized tuning at lower values of the threshold.

The different performance of an active or slim design is shown in Fig.5 for $150 \mu \mathrm{m}$ thick sensors, where it can be noticed that the slim edge design is only partially efficient in the edge 

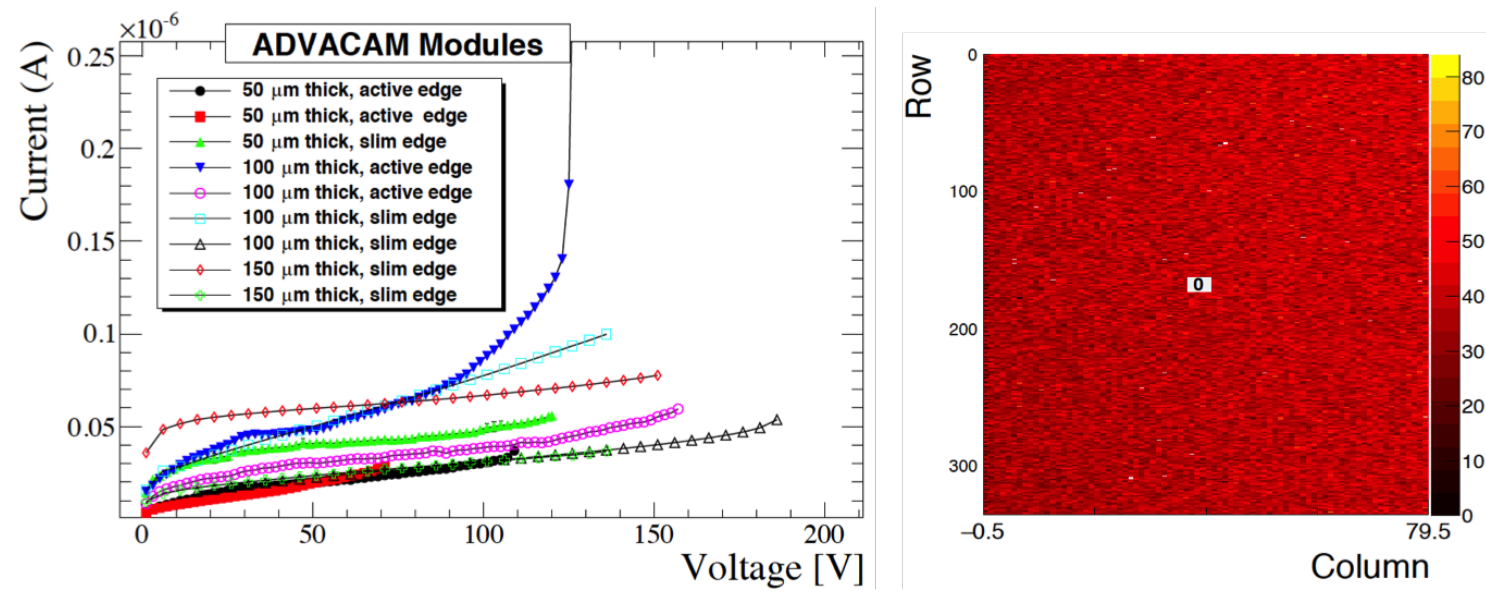

Figure 3. Left: IV curves measured on FE-I4 modules with ADVACAM sensors with a thickness of 50, 100 and $150 \mu \mathrm{m}$. Right: Hit map obtained with a Cadmium source using a $50 \mu \mathrm{m}$ thin sensor interconnected to a FE-I4 chip.

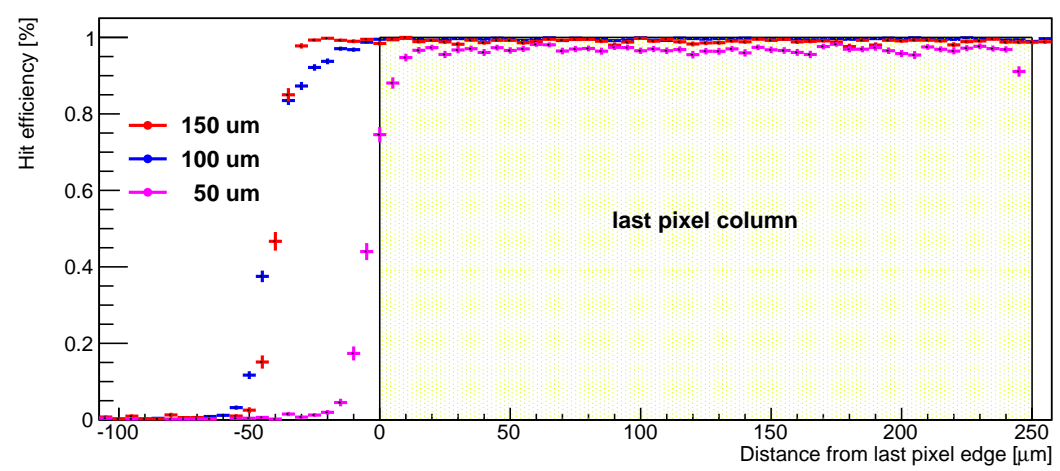

Figure 4. Hit efficiency measured on not irradiated active edge sensors of 50, 100 and $150 \mu \mathrm{m}$ thickness. The values are averaged among the pixels of the edge column. The yellow rectangle indicates the position of the pixel $\mathrm{n}^{+}$implant of the last pixel column terminating at $\mathrm{x}=0$ while the sensor phisically ends at $\mathrm{x}=-50$ $\mu \mathrm{m}$.

region, given the fact that the charge collected by the bias ring, kept at ground potential by the read-out chip through two sets of bumps, is not amplified and further read-out.

The performance of active edge devices has also been studied after irradiation at a fluence of $10^{15} \mathrm{n}_{\mathrm{eq}} / \mathrm{cm}^{2}$ with $24 \mathrm{MeV}$ protons at the MC40 Proton Cyclotron in Birmingham [12], and the corresponding hit efficiency is shown in Fig.6. Increasing the bias voltage up to a value of $250 \mathrm{~V}$, the edge efficiency is fully recovered and also the efficiency loss due to charge sharing between neighbouring pixels, visible in Fig.7 at the four corners of the pixel cell, is partially reduced.

\section{Tracking performance at high incidence angle}

Smaller pixel cells are challenging for the tracking particles in regions of high pseudo-rapidity (high $\eta$ ). To make predictions on the performance of a $50 \times 50 \mu \mathrm{m}^{2}$ pixel cell at high incidence 


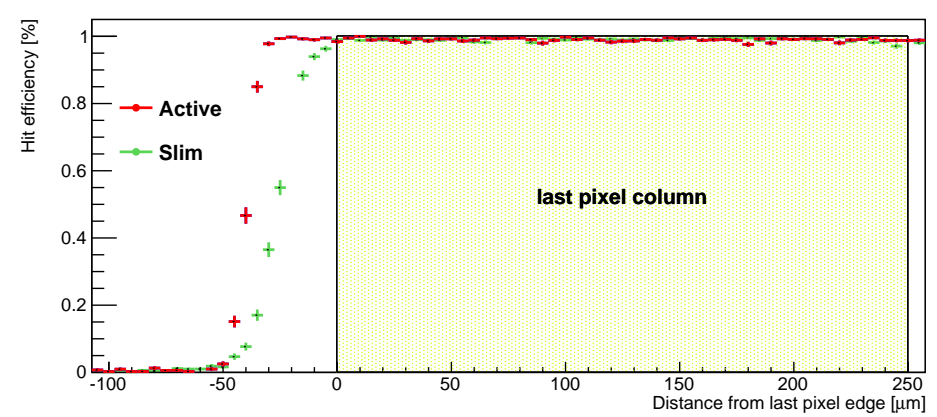

Figure 5. Hit efficiency measured on not irradiated $150 \mu \mathrm{m}$ thick sensors with active or slim edges. The values are averaged among the pixels of the edge column. The yellow rectangle indicates the position of the pixel $\mathrm{n}^{+}$implant of the last pixel column terminating at $\mathrm{x}=0$ while the sensor phisically ends at $\mathrm{x}=-50 \mu \mathrm{m}$ for the active edge design and at $\mathrm{x}=-100 \mu \mathrm{m}$ for the slim edge design.

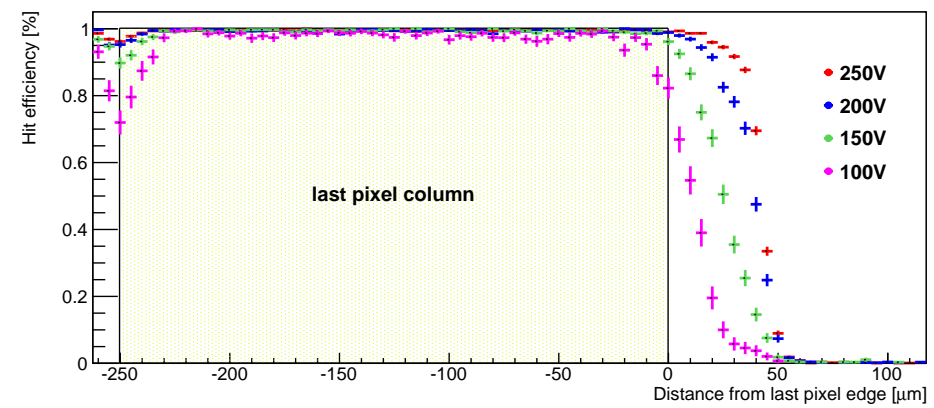

Figure 6. Hit efficiency measured measured with a $150 \mu \mathrm{m}$ thick sensor with active edges after irradiation at a fluence of $10^{15} \mathrm{n}_{\mathrm{eq}} / \mathrm{cm}^{2}$.

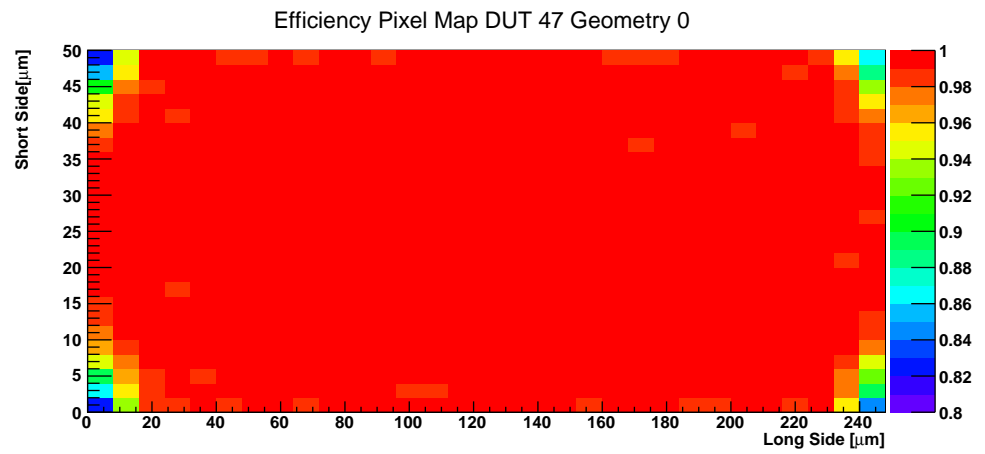

Figure 7. In-pixel hit efficiency map of a $150 \mu \mathrm{m}$ thick sensor with active edges at a bias voltage of $250 \mathrm{~V}$, after irradiation at a fluence of $10^{15} \mathrm{n}_{\mathrm{eq}} / \mathrm{cm}^{2}$.

angle, FE-I4 modules were arranged such that the particles of the beams at DESY and CERN were crossing the pixel along the short side $(50 \mu \mathrm{m})$, at an inclination corresponding to $\eta$ values around 2-2.5. The hit efficiency for each single pixel in the cluster is shown in Fig.8 for sensors of 100, 150 and $200 \mu \mathrm{m}$ thickness. The single hit efficiency is defined as the total number of hits divided by 
the cluster length not including the entrance and exit pixels that are $100 \%$ efficient by construction. All modules were tuned to a threshold of $1000 \mathrm{e}^{-}$. It can be observed that the cluster size depends strongly on the sensor thickness and that the hit efficiency is higher for the thinner sensors, possibly due to the reduced effect of charge sharing for lateral diffusion to the neighbouring pixels.

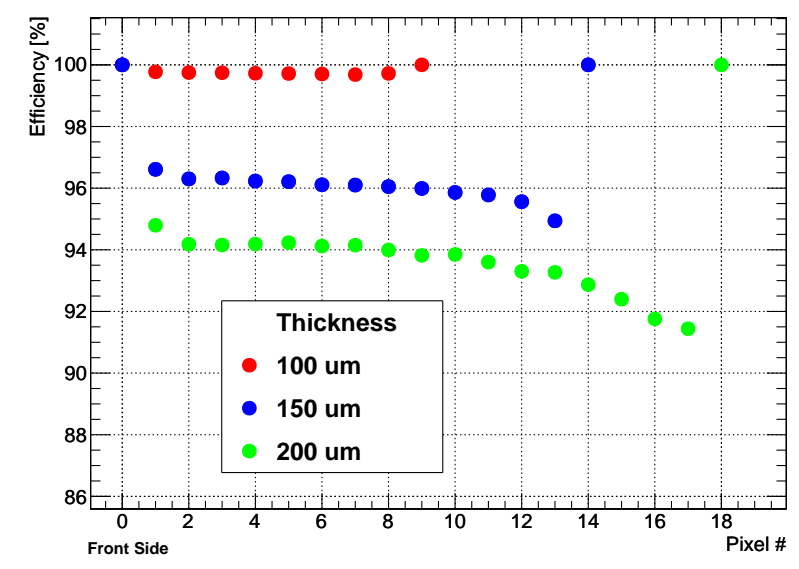

Figure 8. Hit efficiency as a function of pixel number for not irradiated FE-I4 modules with a sensor thickness of 100,150, $200 \mu \mathrm{m}$, inclined by $76-78^{\circ}$ with respect to the beam direction. The efficiencies of the first and last pixels are by construction $100 \%$ since they define the start and the end of the cluster.

\section{Conclusions}

Thin planar n-in-p pixel modules, with sensors in the thickness range 50-150 $\mu \mathrm{m}$, with and without active edges, were investigated in view of the upgrade of the ATLAS pixel system for HL-LHC. Very good interconnection yield between sensors and chips has been observed for these thin devices produced at CiS and ADVACAM. Active edge sensors were investigated in terms of hit efficiency, both in the central active area and in the edge for different designs. The active area has been measured to extend up to the sensor physical perimeter for the design with only a floating guard ring whereas the hit efficiency goes to zero in correspondence to the bias ring in the devices where this structure has been implemented. An active edge sensor was irradiated at a fluence of $10^{15}$ $\mathrm{n}_{\mathrm{eq}} / \mathrm{cm}^{2}$ and increasing the bias voltage up to $250 \mathrm{~V}$, the same tracking performance as in the not irradiated case could be obtained. To allow for an investigation of the hit efficiencies at high pseudo-rapidity at HL-LHC for the $50 \times 50 \mu \mathrm{m}^{2}$ pixel cell, FE-I4 modules were tested in beam tests. The cluster properties were analyzed and a good hit efficiency extracted for the single pixels, with the better performance observed for the thinner sensors, in the thickness range 100-200 $\mu \mathrm{m}$.

\section{Acknowledgments}

This work has been partially performed in the framework of the CERN RD50 Collaboration. The authors would like to thank A. Dierlamm for the irradiation at KIT, V. Cindro for the irradiations at JSI and L. Gonella for the irradiations at the MC40 Cyclotron of the University of Birmingham. Supported by the H2020 project AIDA-2020, GA no. 654168. 


\section{References}

[1] B. Smart on behalf of the ATLAS Collaboration, "ATLAS Pixel Detector Design for HL-LHC", to be published in the same conference proceeding issue.

[2] The ATLAS Collaboration, Technical Design Report for the ATLAS ITk-Strips Detector, in publication.

[3] A. La Rosa, ATLAS Pixel Detector: Operational experience and Run-1 to Run-2 transition. Proceeding of Science PoS(Vertex2014) 001 (2014).

[4] RD53 Collaboration, http://rd53.web.cern.ch/RD53

[5] N. Savic et al., Investigation of thin n-in-p planar pixel modules for the ATLAS upgrade, Proceedings of the 18th IWORID workshop, JINST 11 (2016) C12008 .

[6] CiS Forschungsinstitut für Mikrosensorik GmbH, http://www.cismst.org/

[7] N. Savic et al., Thin n-in-p planar pixel modules for the ATLAS upgrade at HL-LHC, Proceedings of the Vienna Conference on Instrumentation 2016, Nucl. Instr. Meth. A (2016).

[8] Fraunhofer Institute for Reliability and Microintegration IZM, http://www.izm.fraunhofer.de/en.html

[9] http://www.advacam.com/en/

[10] X. Wu et al., Recent advances in processing and characterization of edgeless detectors, JINST 7 (2012) C02001.

[11] H. Jansen et al., Performance of the EUDET-type beam telescopes, EPJ Techniques and Instrumentation, 2016.

[12] http://aida2020.web.cern.ch/content/uob 